\title{
Multivariate Logistic Analysis and Intervention Countermeasures on the Effects of Psychological Changes in Patients with COVID-19 during Treatment
}

\author{
Guangtao $\mathrm{Li}^{1}$, Yaping Wang ${ }^{1}$, Dongxu Zhao ${ }^{2}$, Heyi Zhang ${ }^{1}$, Zhichao Shao ${ }^{1}$, Dan $\mathrm{Ma}^{1}$, Xiqiu Zeng ${ }^{1, *}$ \\ ${ }^{1}$ Respiratory Department, Mudanjiang Kangan Hospital, Mudanjiang 15700, Heilongjiang, China. \\ ${ }^{2}$ Department of Psychiatry, Hongqi Hospital Affiliated to Mudanjiang Medical University, Mudanjiang 15700, Heilongjiang, China.
}

\begin{abstract}
How to cite this paper: Guangtao $\mathrm{Li}$ Yaping Wang, Dongxu Zhao, Heyi Zhang, Zhichao Shao, Dan Ma, Xiqiu Zeng. (2021) Multivariate Logistic Analysis and Intervention Countermeasures on the Effects of Psychological Changes in Patients with COVID-19 during Treatment. International Journal of Clinical and Experimental Medicine Research, 5(4), 460-465.

DOI: 10.26855/ijcemr.2021.10.007
\end{abstract}

Received: August 9, 2021

Accepted: August 31, 2021

Published: September 24, 2021

*Corresponding author: Xiqiu Zeng, Respiratory Department, Mudanjiang Kangan Hospital, Mudanjiang 15700, Heilongjiang, China.

Email: 565651826@qq.com

\begin{abstract}
Objectives: to explore the multivariate Logistic analysis and intervention countermeasures on the influence of psychological changes of COVID-19 patients among returned overseas migrant workers during treatment. Methods: A questionnaire was conducted via the Internet to 100 COVID-19 patients among returned overseas workers who were treated from March 2020 to April 2020. The 100 patients were divided into two groups. After the questionnaire survey, the psychological effects of the 100 patients were evaluated, the clinical data characteristics of the two groups were compared, and the results of multivariate logistic regression model were analyzed. Results: The questionnaire survey data of the 100 COVID-19 patients showed no significant difference in gender, age, permanent residence, average monthly income and education level $(p>0.05)$. Univariate and multivariate Logistic results showed that the incidence of anxiety, depression, loneliness and fear in COVID-19 patients during treatment was not significantly correlated with gender, marital status and medical background $(p>0.05)$, but it was significantly correlated with age, permanent residence, average monthly income and education level ( $p<$ 0.05). Conclusion: Age, permanent residence, average monthly income and education level will affect the psychological state of COVID-19 patients to varying degrees. Consequently, clinicians shall closely observe the psychological changes of COVID-19 patients, and professional psychologists and nurses shall be invited to carry out targeted psychological nursing and intervention for existing problems among patients so as to help COVID-19 patients release their unhealthy emotions. This is of great significance, can promote the effective control of diseases of patients with COVID-19 and is worthy of popularization and application.
\end{abstract}

\section{Keywords}

COVID-19, Multivariate Logistic Analysis, Intervention Countermeasures, Psychological State, Influencing Factor

COVID-19 is a novel infectious disease that can spread among people. In December, 2019, a novel coronary pneumonia broke out in Wuhan, Hubei Province, China. Fever, fatigue, dry cough, respiratory disturbance, etc., 
were the early manifestations of this pneumonia. Droplet transmission and contact transmission were its main transmission ways. COVID-19 can result in respiratory failure, and acute respiratory syndromes and even death to patients. The rapid occurrence of COVID-19, accompanied by serious life threat, has caused great negative anxiety to the psychology of people in various regions of China [1]. Hence, in this paper, 100 COVID-19 patients among returned overseas workers were selected to explore the multivariate Logistic analysis and intervention countermeasures on the influence of psychological changes of COVID-19 patients during treatment.

\section{Materials and Methods}

\subsection{Clinical data}

A questionnaire was conducted via the Internet to 100 COVID-19 patients among returned overseas workers who were treated from March 2020 to April 2020. The 100 patients were divided into two groups. After the questionnaire survey, the psychological effects of the 100 patients were evaluated, the clinical data characteristics of the two groups were compared, and the results of multivariate logistic regression model were analyzed.

\subsection{Inclusion and exclusion criteria}

Inclusion criteria: (1) Returned overseas workers; (2) COVID-19 nucleic acid showed positive by real-time fluorescence RT-PCR of respiratory tract specimens; (3) fever or respiratory symptoms; and (4) approved by hospital ethics committee and agreed by patients. Exclusion criteria: (1) patients with abnormal neurological function, abnormal cognitive function or incomplete admission information; (2) those who failed to be admitted to hospital normally due to various reasons; and (3) those who failed to cooperate in the treatment.

\subsection{Methods}

The members of the medical team introduced the purpose and significance of the survey, guided the patients to finish the questionnaire survey and filled out the questionnaire online by using the Internet WeChat platform (the patients completed the questionnaire according to their own situation, including, Self-Rating Anxiety Scale, SAS, and Self-Rating Depression Scale, SDS). SDS scale was first prepared by ZUNG in 1965, which has good validity. The scale included 20 items and each item was evaluated by level-4 scoring methods of 1-4 points, with 50 points as the boundary. No depression for those $<50$ points, minor depression for those at 50-60 points, moderate depression for those at 60-70 points, severe depression for those $>70$ points, and the Cronbach $\alpha$ coefficient of total scale was 0.803 . SAS scale was also divided by 50 points. It was normal for those $<50$ points, minor depression for those at 50-60 points, moderate depression for those at 61-70 points, severe depression for those $>70$ points, and the Cronbach $\alpha$ coefficient of total scale was 0.795 . The members carefully explained the survey to the patients with low education level and difficulty in understanding the contents of the questionnaire and then guided them to complete the questionnaire. The first part of the questionnaire covers the general information of patients, including gender, age, permanent residence, average monthly income, educational level, marital status and medical background, and the second part covers the general mental state scale, including changes of four dimensions, namely, anxiety, depression, loneliness and fear. Each dimension has four items, ranging from "never, seldom" to "most time, all time", and each item is assigned with 1-4 points, respectively. The total score of the questionnaire is 100 points, and the full score of the dimension is 25 points. If the dimension score is $\geq 20$ points, a psychological problem is determined at present and the count is "YES"; if the dimension score is $<20$ points, no psychological problem is determined at present and the count is "NO". The members entered the edited instructions and test questions into the survey questionnaires in turn and then send them to the Internet WeChat platform. Based on the survey results, statistical analysis was carried out to analyze the influencing factors of psychological changes of COVID-19 patients during treatment [2-3].

\subsection{Observation target}

(1) Analysis of general clinical data of COVID-19 patients who received the treatment, mainly from the aspects of gender, age, permanent residence, average monthly income, education level, marital status and medical background, etc [4-5].

(2) Comparison of psychological status of patients with different characteristics. The incidence of anxiety, depression, loneliness and fear of patients during treatment was compared and analyzed from the aspects of gender, age, permanent residence, average monthly income, education level, marital status and medical background.

(3) Multivariate Logistic analysis of psychological effect of COVID-19 patients during treatment. It was ana- 
lyzed from five aspects, namely, age, average monthly income, permanent residence, educational level and occupation.

\subsection{Statistical analysis}

The data were processed using Software SPSS18.0. The $\chi^{2}$ test was used for the counting data, which was expressed by $n$ (\%); the $t$ test was used for the measuring data, which was expressed by $\overline{\mathrm{x}} \pm$ s. $p<0.05$ means that the difference is statistically significant.

\section{Result}

\subsection{Analysis of general information of $\mathbf{1 0 0}$ patients}

The questionnaire survey data of COVID-19 patients showed no significant difference in gender, age, permanent residence, average monthly income, education level, marital status and medical background ( $p>0.05)$, as shown in Table 1.

Table 1. Analysis of General Clinical Data of 100 Treated Patients

\begin{tabular}{|c|c|c|c|c|}
\hline & Clinical Data & Cases $(n=100)$ & $\chi^{2}$ & $p$ \\
\hline \multirow{2}{*}{ Gender } & Male & $54(54.00)$ & \multirow{2}{*}{1.302} & \multirow{2}{*}{0.143} \\
\hline & Female & $46(46.00)$ & & \\
\hline \multirow[t]{3}{*}{ Age } & $\geq 60$ years old & $67(67.00)$ & \multirow{3}{*}{0.681} & \multirow{3}{*}{0.139} \\
\hline & 21-59 years old & $23(23.00)$ & & \\
\hline & $\leq 20$ years old & $10(10.00)$ & & \\
\hline \multirow[t]{2}{*}{ Permanent residence } & City & $54(54.00)$ & \multirow{2}{*}{0.993} & \multirow{2}{*}{0.072} \\
\hline & Countryside & $46(46.00)$ & & \\
\hline \multirow[t]{4}{*}{ Average monthly income } & $\leq 3,000$ Yuan & $26(26.00)$ & \multirow{4}{*}{1.395} & \multirow{4}{*}{0.071} \\
\hline & $3,000-5,000$ & $32(32.00)$ & & \\
\hline & $5,001-10,000$ & $22(22.00)$ & & \\
\hline & $>10,000$ & $20(20.00)$ & & \\
\hline \multirow{3}{*}{ Education level } & Primary school or below & $35(35.00)$ & \multirow{3}{*}{1.591} & \multirow{3}{*}{0.121} \\
\hline & Middle school-undergraduate education & $35(35.00)$ & & \\
\hline & Undergraduate education or above & $30(30.00)$ & & \\
\hline \multirow[t]{2}{*}{ Marital status } & YES & $53(53.00)$ & \multirow{2}{*}{0.923} & \multirow{2}{*}{0.171} \\
\hline & NO & $47(47.00)$ & & \\
\hline \multirow[t]{2}{*}{ Medical background } & YES & $54(54.00)$ & \multirow{2}{*}{0.993} & \multirow{2}{*}{0.072} \\
\hline & NO & $46(46.00)$ & & \\
\hline
\end{tabular}

\subsection{Comparison of psychological status of patients with different characteristics}

Univariate results showed that the incidence of anxiety, depression, loneliness and fear in COVID-19 patients during treatment was not significantly correlated with gender, marital status and medical background $(p>0.05)$, but it was significantly correlated with age, permanent residence, average monthly income and education level $(p<$ $0.05)$, as shown in Table 2.

\subsection{Multivariate Logistic analysis of psychological effects of COVID-19 patients during treatment}

Multivariate results showed that the psychological influencing factors of COVID-19 patients during treatment were significantly correlated with age, permanent residence, average monthly income, education level, and occupation $(p<0.05)$, as shown in Table 3. 
Table 2. Psychological status of patients with different characteristics [n (\%)]

\begin{tabular}{|c|c|c|c|c|c|c|c|}
\hline \multicolumn{2}{|c|}{ Clinical data $(n=100)$} & \multirow{3}{*}{$\begin{array}{c}\text { Anxiety } \\
30(30.00) \\
27(27.00)\end{array}$} & \multirow{3}{*}{$\begin{array}{l}\text { Depression } \\
48(48.00) \\
50(50.00)\end{array}$} & \multirow{3}{*}{$\begin{array}{l}\text { Loneliness } \\
54(54.00) \\
46(46.00)\end{array}$} & \multirow{3}{*}{$\begin{array}{c}\text { Fear } \\
24(24.00) \\
26(26.00)\end{array}$} & \multirow{3}{*}{$\frac{\chi^{2}}{1.302}$} & \multirow{3}{*}{$\frac{p}{0.143}$} \\
\hline \multirow{2}{*}{ Gender } & Male & & & & & & \\
\hline & Female & & & & & & \\
\hline \multirow[t]{3}{*}{ Age } & $\geq 60$ Years old & 37 (37.00) & 39 (39.00) & 47 (47.00) & 45 (45.00) & & \\
\hline & 21-59 Years old & $13(13.00)$ & $10(10.00)$ & $15(15.00)$ & $8(8.00)$ & 5.681 & 0.039 \\
\hline & $\leq 20$ Years old & $26(26.00)$ & $20(20.00)$ & $40(40.00)$ & 38 (38.00) & & \\
\hline \multirow[t]{2}{*}{$\begin{array}{l}\text { Permanent } \\
\text { residence }\end{array}$} & City & $14(14.00)$ & $12(12.00)$ & $9(9.00)$ & $11(11.00)$ & 6.993 & 0.022 \\
\hline & Countryside & 37 (37.00) & 27 (27.00) & 47 (47.00) & 32 (32.00) & & \\
\hline \multirow{4}{*}{$\begin{array}{l}\text { Average } \\
\text { monthly } \\
\text { income }\end{array}$} & $\leq 3,000$ Yuan & $66(66.00)$ & $57(57.00)$ & $54(54.00)$ & $46(46.00)$ & & \\
\hline & $3,000-5,000$ & $42(42.00)$ & $32(32.00)$ & $42(42.00)$ & $32(32.00)$ & 5.395 & 0.041 \\
\hline & $5,001-10,000$ & $22(22.00)$ & $12(12.00)$ & $23(23.00)$ & $10(10.00)$ & & \\
\hline & $>10,000$ & $6(6.00)$ & $6(6.00)$ & $9(9.00)$ & $5(5.00)$ & & \\
\hline \multirow{3}{*}{$\begin{array}{l}\text { Education } \\
\text { level }\end{array}$} & $\begin{array}{l}\text { Undergraduate edu- } \\
\text { cation or above }\end{array}$ & $65(65.00)$ & $54(54.00)$ & $57(57.00)$ & $53(53.00)$ & & \\
\hline & $\begin{array}{c}\text { Middle } \\
\text { school-undergraduate } \\
\text { education }\end{array}$ & $45(45.00)$ & $40(40.00)$ & 35 (35.00) & 37 (37.00) & 6.591 & 0.021 \\
\hline & $\begin{array}{l}\text { Primary school or } \\
\text { below }\end{array}$ & $22(22.00)$ & $12(12.00)$ & $23(23.00)$ & $10(10.00)$ & & \\
\hline \multirow[t]{2}{*}{$\begin{array}{c}\text { Marital } \\
\text { status }\end{array}$} & YES & $30(30.00)$ & $48(48.00)$ & $54(54.00)$ & $24(24.00)$ & 1.395 & 0.241 \\
\hline & NO & 27 (27.00) & $50(50.00)$ & $46(46.00)$ & $26(26.00)$ & & \\
\hline \multirow[t]{2}{*}{$\begin{array}{c}\text { Medical } \\
\text { background }\end{array}$} & YES & $30(30.00)$ & $48(48.00)$ & $53(53.00)$ & $20(20.00)$ & 1.395 & 0.141 \\
\hline & NO & 27 (27.00) & $50(50.00)$ & $42(42.00)$ & $22(22.00)$ & & \\
\hline
\end{tabular}

Table 3. Multivariate Logistic Analysis of Psychological Effects of COVID-19 Patients during Treatment

\begin{tabular}{ccccccc}
\hline Multivariate & $\beta$ value & S.E & Wald & $p$ value & OR value & $95 \%$ CI \\
\hline Age & 1.215 & 0.321 & 8.336 & 0.000 & 3.591 & $2.491-6.324$ \\
Average monthly income & 1.131 & 0.093 & 8.111 & 0.000 & 4.574 & $1.496-9.481$ \\
Permanent residence & 1.573 & 0.036 & 9.437 & 0.000 & 6.413 & $5.691-8.461$ \\
Education level & 1.442 & 0.074 & 6.324 & 0.000 & 4.091 & $3.235-4.573$ \\
Occupation & 0.747 & 0.015 & 3.295 & 0.000 & 5.326 & $4.395-7.893$ \\
\hline
\end{tabular}

\section{Discussion}

COVID-19 is a virus widely existing in nature and it is named because its morphology is similar to a crown under electron microscope [6]. Up to now, it has been found that the coronavirus only infects spinal animals and can cause diseases of respiratory tracts, digestive tracts and nervous systems of human beings and animals. At the end of 2019, pneumonia occurred in patients in Wuhan due to unknown reasons, and a novel coronavirus was isolated from the lower respiratory tract of patients, which was named COVID-19 by the World Health Organization (WHO). Fever and fatigue were its main clinical manifestations, and followed by dry cough in respiratory symptoms [7-8]. A few patients were accompanied by nasal congestion, runny nose, discomfort throat, muscle soreness, diarrhea and other symptoms, and dyspnea or hypoxemia gradually happened. Severe patients, especially the elder- 
ly patients and patients with chronic basic diseases, may have acute respiratory distress syndrome, septic shock, heart failure, renal failure, metabolic acidosis difficult to be corrected and coagulation dysfunction. Mild patients have a good prognosis result, and a few patients are in critical conditions or even die, which may cause bad psychological state to NCP patients and medical staff. It is particularly important to take a series of intervention measures to relevant personnel [9-10].

The pneumonia infected by novel coronavirus were suddenly broke out in some patients. In the face of unknown viruses, the psychological state of different people fluctuated in different degrees. The pneumonia infected by novel coronavirus develops rapidly and can threaten the life safety of people. With the rapid change of daily data and less understanding of the novel coronavirus, or family members and relatives staying the epidemic areas, different degrees of psychological stress responses may occur, which is a normal phenomenon. The novel coronavirus is better understood and the epidemic situation is controlled during the development of the epidemic situation, so the psychological stress is gradually alleviated. In case of an existence of psychological fluctuation all the time, it may cause social instability and serious adverse consequences [11-12]. In this study, the psychological changes of COVID-19 patients during treatment were investigated. Univariate results showed that the incidence of anxiety, depression, loneliness and fear in COVID-19 patients during treatment was not significantly correlated with gender, marital status and medical background ( $p>0.05$ ), but it was significantly correlated with age, permanent residence, average monthly income and education level of patients $(p<0.05)$. Most patients are between 30 and 50 years old. Most of them are the pillars of their families and the society and bear more family and social responsibilities. Hospitalization and isolation will have a certain impact on their study, life and work, and they will also fear for that the incurable disease will affect their future life and bring a burden to their families [13-14]. Moreover, different permanent residence places of patients will also lead to different psychological fluctuations. The incidence of anxiety, depression, loneliness and fear of patients living in cities is higher than that of patients living in rural areas, which may be associated with the faster economic development of cities, higher living standards of cities, greater living pressure of residents, huge differences between urban and rural development in China, profound differences in ideological and cultural level and uneven sampling between urban and rural regions. This study also revealed that the education level is the reason that affects the unhealthy psychological phenomenon of patients with COVID-19, which may be related to their better understanding of the disease. They are more aware of the serious infectivity and danger of COVID-19, more sensitive to the surrounding environment, and more anxious about the future uncertainty and the uncertainty of this disease itself. For this reason, it is suggested that the medical staff shall adopt targeted counseling methods for different psychological problems in patients diagnosed with COVID-19 to alleviate and release the negative emotions [15]. Multivariate results showed that the psychological influencing factors of COVID-19 patients during treatment were significantly correlated with age, permanent residence, education level, and other factors $(p<0.05)$. The study results revealed that the average monthly income will lead to different degrees of bad psychological state in COVID-19 patients. After the discharge from hospital, these patients can no longer go abroad to work, and they can only look for jobs in their permanent residence places. Among them, patients with an average monthly income of less than RMB 3,000 Yuan have a higher incidence of unhealthy psychology state, and the low income causes the patients to feel worried and anxious about future treatment expenditure and normal life in the future. Consequently, medical staff shall pay close attention to the psychological changes of COVID-19 patients, conduct targeted interventions on the adverse psychological state of different patients, actively mobilize the social support system, encourage family members, friends and colleagues of patients to contact patients actively through online communication methods like WeChat, and encourage them to make active cooperation during the treatment and keep an optimistic attitude to face the disease.

In conclusion, age, permanent residence, average monthly income and education level of patients will affect the psychological state of COVID-19 patients to varying degrees. Hence, clinicians shall closely observe the psychological changes of COVID-19 patients, and professional psychologists and nurses shall be invited to carry out targeted psychological nursing and intervention for existing problems among patients so as to help COVID-19 patients release their unhealthy emotions. This is of great significance, can promote the effective control of diseases of patients with COVID-19 and is worthy of popularization and application.

\section{References}

[1] Nan, L., Fang, Q. Y., and Liu, D. (2018). Logistic regression analysis of risk factors of ventilator-associated pneumonia in ICU patients and intervention effect analysis. Journal of Clinical Emergency, 2018, vol.19, No. 143(05): 44-48. 
[2] Zhang, H., Fang, Q., Hu, J. X., et al. (2019). Study on influencing factors and preventive measures for multi-drug resistant Klebsiella pneumonia infection in ICU patients. Chinese Journal of Nosocomiology, 2019, 29(02): 48-50+55.

[3] Liu, H., Xu, H., Zhang, X. L., et al. (2019). Logistic regression analysis for evolution law and influencing factors of phlegm-heat closed lung syndrome in children with pneumonia and asthma cough. Chinese Journal for Clinicians, 2019, 47(01): 121-124.

[4] Li, H. Y., Guo, Q., Song, W. D., et al. (2018). Priority for Treatment and Intensive Care of Patients With Non-Severe Community-Acquired Pneumonia. American Journal of the Medical Sciences, 2018, 356(4): 329-334.

[5] Kang, Y., Fang, X. Y., Hou, Y. P., et al. (2018). Relationship between Nutritional Status and Severity of Pneumonia in Elderly Patients with Community-acquired Pneumonia. Chinese General Practice, 2018, 21(28): 86-90.

[6] Wang, Y., Yang, Y. Y., Li, S. W., et al. (2020). Investigation on depression of children and adolescents at home during the period of COVID-19 epidemic situation and analysis of its influencing factors. Chinese Journal of Child Health Care, 2020, 28(03): 277-280.

[7] Cheng, L., Zheng, L. P., Yan, S. Y., et al. (2020). Anxiety status quo of COVID-19 patients and analysis of its influencing factors. Zhejiang Medicine, 2020, 42(4): 315-317.

[8] Chang, J. H., Yuan, Y. X., and Wang, D. (2020). Mental health status of college students during COVID-19 epidemic situation and analysis of its influencing factors. Journal of Southern Medical University, 2020, 40(02): 171-176.

[9] Cheng, J. G., Tan, X. D., Zhang, L., et al. (2020). Study on the influencing factors of psychological status of confirmed COVID-19 patients and isolated patients. Journal of Nursing Administration, 2020, 20(04): 247-251.

[10] Lu, D., Wang, H., Yu, R., et al. (2020). Integrated infection control strategy to minimize nosocomial infection of coronavirus disease 2019 among ENT healthcare workers. Journal of Hospital Infection, 2020, 104(4): 454-455.

[11] Liu, J., Yu, H., and Zhang, S. (2019). The indispensable role of chest CT in the detection of coronavirus disease 2019 (COVID-19). European Journal of Nuclear Medicine and Molecular Imaging, 2020, 47(7): 1638-1639.

[12] Tan, W., Hu, B. Z., Zhang, Z., et al. (2020). Clinical features and analysis of death factors of severe novel coronavirus pneumonia. Chinese Journal of Tuberculosis and Respiratory Diseases, 2020, 43(00): E048-E048

[13] Sun, D. W., Zhang, D., Tian, R. H., et al. (2020). Analysis of influencing factors of liver function damage in novel coronavirus pneumonia and its clinical significance. Chinese Journal of Digestive Surgery, 2020, 19(04): 360-365.

[14] Liu, C. L., Yuan, Y. L., Wang, J. Y., et al. (2019). Current status and influencing factors of ventilator-associated pneumonia in comprehensive intensive care unit. West China Medical Journal, 2019, 034(008): 907-911.

[15] Feng, Y., Tang, Z. P., Nie, D. Y., et al. (2018). Analysis of the risk factors and pathogenic characteristics of ventilator-associated pneumonia in intensive care unit. People’s Military Surgeon, 2018, 061(005): 412-415. 